\title{
Beurteilung von Synergiepotenzialen am Beispiel der Megafusion von AOL und Time Warner
}

\author{
Sascha L. Schmidt / Patrick Vogt
}

Die Fusion von AOL und Time Warner zum weltweit größten integrierten Medien-, Unterhaltungs- und Internetkonzern war nicht nur der größte Merger in der Wirtschaftsgeschichte, sondern auch einer der umstrittensten. Die Autoren analysieren die Fusion anhand zweier theoretischer MEA-Ansätze mit Fokus anf die Perspektive des Unternebmens, der so genannten Capital Market School sowie der Strategic Management School. Aus beiden Perspektiven wurde die Fusion vorab positiv eingeschätzt. Die Autoren zeigen jedoch unter Rückgriff auf die Strategieprozessforschung, dass weder eine Kapitalmarktabschätzung noch die Ermittlung des strategischen Fits der beiden Mergerpartner ausreichte, um die Erfolgswahrscheinlichkeit des Zusammenschlusses ex ante einschätzen zu können. Eine wichtige Rolle dafür spielten Probleme bei Entwicklung und Umsetzung der Strategie für den Merger, aber auch die Branchen der Mergerpartner. Ein Vergleich mit Zusammenschlïssen äbnlicher Größenordnung in anderen Branchen zeigt darüber hinaus generelle Probleme von Megafusionen auf. Die Autoren schlagen vor, neben dem Synergiepotenzial auch einen Faktor für die Realisierbarkeit des Mergerkonzepts zu ermitteln, mit dessen Hilfe die Erfolgswahrscheinlichkeit einer $M E A-T r a n s a k t i o n$ validiert werden kann. In diesem Zusammenhang kommt der Analyse der Kulturkompatibilität der Mergerpartner große Bedeutung zu.

Stichwörter: AOL, TimeWarner, Merger, Fusion, Capital Market School, Strategic Management School, Post Merger Management, Strategieimplementierung, Synergien, Medienindustrie

\section{Einleitung}

Der Zusammenschluss von AOL und Time Warner zum weltweit größten integrierten Medien-, Unterhaltungs- und Internetkonzern beschäftigt seit gut vier Jahren Wissenschaftler, Aktionäre, Analysten und Medienexperten gleichermaßen. Dafür sind verschiedene Gründe verantwortlich: Erstens handelte es sich um die größte Übernahme in der US-amerikanischen Wirtschaftsgeschichte. Zweitens erzeugte die Tatsache, dass die gemäß Ertrag und Anzahl der Mitarbeiter größere Unternehmung (Time Warner) von der kleineren (AOL) übernommen wurde, große Aufmerksamkeit. Zwar verfügte AOL zur Zeit des Mergers über eine weitaus größere Marktkapitalisierung. Gemessen an Ertrag und Anzahl der Mitarbeiter war das Verhältnis zwischen AOL und Time Warner aber etwa $1: 5 .{ }^{1}$ Drittens war der Unternehmenszusammenschluss mit außerordentlich hohen Erwartungen verknüpft. Entsprechend stark waren die negativen Reaktionen auf die Nichterfüllung dieser Erwartungen.

Es ist auffallend, dass die anfänglich äußerst positive Beurteilung des Mergers von ei-

1 Die Erträge von AOL beliefen sich auf 18 Prozent im Vergleich zu Time Warner (15 Prozent der kumulierten Erträge von AOL und Time Warner im Jahre 1999), die Anzahl der Mitarbeiter von AOL war 20 Prozent im Vergleich zu Time Warner (17 Prozent der kumulierten Mitarbeiterzahl im Jahr 1999) (Time Warner 2000a: 41/45; AOL 2000: 17). 
nem Extrem ins andere überging. Entsprechend findet in der Fachpresse wie in der Wissenschaft eine teilweise polemisch gefärbte Auseinandersetzung anstelle einer nüchternen Analyse der Tatbestände statt (bspw. Peers/Brown 2003: 1; Munk 2003: 21; Peers/Angwin 2003a: 1, aber auch Klein 2003). In diesem Artikel soll nun eine systematische Analyse der Fusion von AOL und Time Warner und eine Bewertung vor dem Hintergrund von zwei theoretischen Ansätzen aus der M\&A-Forschung vorgenommen werden (M\&A bezeichnet im Folgenden „Mergers and Acquisitions“, also Fusionen und Übernahmen von Unternehmen). Es soll aufgezeigt werden, inwieweit Integrationsprobleme die ursprüngliche Einschätzung des Synergiepotenzials verändert haben und welche spezifischen Anforderungen die Formulierung eines gemeinsamen Mergerkonzepts für die Mergerpartner grundsätzlich mit sich bringt. Es sollen weiter Lösungswege aufgezeigt werden, um die Beurteilung des Erfolgspotenzials eines Mergers ex ante zu erleichtern mit dem Ziel, einen häufig zu beobachtenden Post Merger Drift zu verhindern. Schließlich soll aufgezeigt werden, welche Besonderheiten einem Merger in der Medienindustrie zukommen.

Theoretische M\&A-Ansätze werden zur Analyse herangezogen, um zu einer fundierten und objektiven Einschätzung eines Mergers zu gelangen. Sie ermöglichen die Beurteilung eines Mergers aus unterschiedlichen Perspektiven bzw. den Blick durch unterschiedliche „Linsen“. Aus ökonomischer Sicht sind zwei Ansätze zu unterscheiden: die Capital Market School (kurzfristig, kapitalmarktorientiert) und die langfristig, volkswirtschaftlich orientierte Industrial Organization School (Böckli 2003: 81-123). Verhaltenswissenschaftliche Ansätze (Organization Behavior School) fragen nach dem Einfluss von Persönlichkeitsmerkmalen des Top-Managements auf den Mergererfolg und nach dem Einfluss von M\&A-Transaktionen auf die Mitarbeiter (Haspeslagh/Jemison 1991: 293-306). Ansätze des strategischen Managements (Strategic Management School) schließlich berücksichtigen den Einfluss unternehmensinterner Vorgänge während der Integrationsphase auf den Mergererfolg. Maßnahmen, welche auf Entscheidungen des Managements beruhen, werden als primäre Quellen der Wertschöpfung während der Integration betrachtet (Larsson/Finkelstein 1999: 5).

Im Folgenden wird die Fusion von AOL und Time Warner aus Kapitalmarktsicht sowie aus strategischer Managementsicht analysiert. Damit sollen zum einen diejenigen Ansätze herangezogen werden, welche sich auf die Sicht des Unternehmens fokussieren, zum anderen soll die kurzfristige kapitalmarktorientierte mit der langfristigen, unternehmerischen Perspektive des Zusammenschlusses kontrastiert werden.

\section{Die Fusion von AOL und Time Warner}

Die Ankündigung der Übernahme von Time Warner durch AOL am 10. Januar 2000 hat in der Medienindustrie große Aufmerksamkeit erzeugt. Mit AOL übernahm das weltweit größte Internet-Unternehmen den weltweit größten Medien- und Unterhaltungskonzern, Time Warner. Damit gelang es einem Internet-Unternehmen während des Internetbooms und, wie sich später herausstellen sollte, rund zwei Monate vor dem Platzen der Internet-Blase, ein nachhaltiges, ertragsstarkes und verwandtes Geschäft zu übernehmen. Zwar betonten die beiden Architekten der Transaktion, Steve Case, Gründer, Chairman und CEO von AOL, und Gerald Levin, Chairman und CEO von Time Warner, es handle sich um eine Fusion gleicher Partner, d. h. um einen so genannten „Merger of Equals“ (Levin/Case 2000: 7). Trotzdem wurde der Zusammenschluss in der Öffentlichkeit (bspw. Waters 2000b: 22; Klinkenborg 2000: 32) gemäß der formellen Transaktion (Time Warner 2000b: 3) als Übernahme von Time Warner 
durch AOL gewertet. ${ }^{2}$ Die Aktionäre von AOL erhielten $55 \%$ der Aktien des neuen Unternehmens.

AOL wurde 11 Jahre, ${ }^{3}$ Time Warner 78 Jahre vor dem Merger gegründet. Time Warner ging 1989 aus der Fusion von Time Inc. und Warner Communications ${ }^{4}$ hervor und war bereits zu diesem Zeitpunkt das weltweit führende Medienhaus. Sechs Jahre später, 1995, wurde Turner Broadcasting Systems mit dem Flagschiff CNN übernommen und integriert. ${ }^{5}$ Time Warner war einer traditionellen, journalistischen Kultur verpflichtet und operierte immer noch von den beiden ehemaligen Hauptsitzen von Time Inc. und Warner aus. ${ }^{6}$ Das Unternehmen fokussierte sich auf Geschäftsfelder, welche journalistische Kompetenz, Kreativität und Innovativität erforderten. Dementsprechend verfügte Time Warners Geschäftsportfolio z. B. über das Hollywood-Filmstudio Warner Bros. und eines der weltgrößten Musiklabel, Warner Music Group (Grimes 2001: 15).

AOL hingegen wurde vor allem durch das Kerngeschäft des so genannten Internet Service Providing (ISP) geprägt, welches sich zu einem Commodity Business entwickelte. Dementsprechend wurden Wettbewerbsvorteile primär durch Kostenvorteile erzielt (Sjurts 2000: 130). AOL entsprach dieser Anforderung durch eine kostenfokussierte, gewinn- und technologieorientierte Ausrichtung mit aggressivem Marktauftritt. Time Warner verfügte hingegen eher über eine umsatzorientierte, auf Produktinnovationen (Sjurts 2000: 131) und konservative Werte ausgerichtete Unternehmenskultur. AOL übernahm zur Stärkung des Kerngeschäfts ISP-Anbieter wie Advanced Networks E Services, Wettbewerber wie CompuServe oder Netscape sowie Inhalte-Anbieter wie Spinner.com, ein Internet-Musik Start-up etc. (Strunck 2003: 215ff.). Abbildung 1 zeigt die zentralen Fakten zu AOL und Time Warner zum Zeitpunkt der Fusions-Ankündigung.

Das Interesse der Medienindustrie war besonders hoch, da es sich bei dem Merger um eine Kombination von „alten“ und „neuen“ Medien handelte. Entstehen sollte ein konvergenter, vollintegrierter Medien-, Unterhaltungs- und Internetkonzern, der die gesamte Wertschöpfungskette abdeckt: Von der Content Creation über das Content Packaging bis zur Content Delivery in Film, Musik, TV, Magazine, Zeitung, Buch und Internet. Unter der Annahme, dass traditionelle Medienunternehmen ohne Internet-Zugang nur bescheidene Entwicklungschancen besitzen, wurde es von zahlreichen Analysten als notweniger Schritt gesehen, ins Internet-Geschäft zu diversifizieren (Levin 2000: 3ff.). Die Fusion von AOL und Time Warner wurde dementsprechend als logische Konsequenz der Marktentwicklung gesehen. Andere Stimmen gingen sogar noch weiter und interpretierten den Zusammenschluss bereits als „Tod der alten Medien“ (Huber 2000: 26).

2 Aufgrund der Eigendeklaration der Mergerpartner werden im Folgenden die Begriffe Merger bzw. Fusion für den Zusammenschluss von AOL Time Warner verwendet.

3 Steve Case und Jim Kinsey, die beiden Gründer von AOL, waren bereits vier Jahre zuvor im Internetgeschäft tätig gewesen. Unter dem Namen Quantum Computer Services vertrieben sie Online-Services wie News, Games etc. an Geschäftskunden (Strunck 2003: 214).

4 Beide Unternehmensteile hatten wiederum ihrerseits einen bemerkenswerten M\&A Track Record in der Vergangenheit (Strunck 2003: 218).

5 „Die Akquisitionskosten von USD 14 Mrd. waren jedoch eine erhebliche Belastung für das Unternehmen. Die Schuldenhöhe stieg auf USD 11 Mrd. Es dauerte über sieben Jahre, bis sich Time Warner von der finanziellen Belastungen der Integration erholt hatte" (Strunck 2003: 219).

6 Beobachter behaupten sogar, die Fusion von Time Inc. und Warner hätte gar nie richtig funktioniert. Sie sei „a textbook example of culture clash. Indeed, people within the company say the integration of the tanned Hollywood set from Warner Bros. and the horn-rimmed Easterners of Time never quite worked out" (Grimes 2001: 15). 
Abbildung 1: Profile von AOL und Time Warner zum Zeitpunkt der Fusionsankündigung

\section{GAOL TimeWarner}

\begin{tabular}{|c|c|c|}
\hline Umsatz (1999) & - USD 4,8 Mrd. & - USD 27,3 Mrd. \\
\hline Marktkapitalisierung & • USD 165 Mrd. & - USD 83 Mrd. \\
\hline Langfristiger & & \\
\hline Schuldenstand & - USD 0,6 Mrd. & - USD 31,9 Mrd. \\
\hline Eigentümer & $\begin{array}{l}\text { - Streubesitz, keine } \\
\text { größeren Aktionäre }\end{array}$ & $\begin{array}{l}\text { - Steubesitz, größte Aktionäre Ted Turner } \\
\text { und John Malone/Liberty Media mit je 10\% }\end{array}$ \\
\hline Leitung & - Stephen M. Case & - Gerald M. Levin \\
\hline Mitabeiter (FTE) & - 12.000 & - 59.000 \\
\hline Gründung & $\begin{array}{l}\text { - } 1985 \text { als Quantum } \\
\text { Computer Services, } 1991 \\
\text { umbenannt in AOL }\end{array}$ & $\begin{array}{l}\text { - } 1989 \text { mit der Fusion der beiden } \\
\text { Traditionsunternehmen Warner } \\
\text { Communication und Time Inc. } \\
\text { (Erste Ausgabe des Time Magazine } \\
\text { datiert von 1923) }\end{array}$ \\
\hline $\begin{array}{l}\text { Wichtigste } \\
\text { Tätigkeitsgebiete }\end{array}$ & $\begin{array}{l}\text { B2C-Internet: 90\% (u.a. } \\
\text { AOL) }\end{array}$ & $\begin{array}{l}\text { - Film: } 28 \% \text { (u.a. Warner Bros.) } \\
\text { - TV: } 21 \% \text { (u.a. CNN-Gruppe, HBO) } \\
\text { - Kabel-TV: } 19 \% \text { (u.a. Time Warner Cable) }\end{array}$ \\
\hline
\end{tabular}

\section{Die Fusion von AOL und Time Warner aus Kapitalmarktsicht}

Aus Kapitalmarktsicht ist für die Beurteilung eines Mergers bedeutsam, ob die Ankündigung des Zusammenschlusses überraschend erfolgt oder nicht. Im letzteren Fall ist davon auszugehen, dass Insider bereits einen Teil des Wertes der Transaktion abgeschöpft haben. Dies war beim Merger von AOL und Time Warner nicht der Fall, die Ankündigung erfolgte überraschend, wie die Entwicklung der Aktienkurse im Vorfeld der Ankündigung verdeutlichte. Time-Warner-Aktien zeigten keine markanten Veränderungen. Die Aktie von AOL stieg zwar im Jahr 1999 im Sog mit anderen Internetwerten stetig, jedoch mit sehr volatilen Ausschlägen nach oben und unten. In den Tagen vor der Ankündigung des Mergers war der Aktienkurs rund 25 Prozent von seinem Vorjahreshöchst entfernt. Abbildung 2 zeigt die Entwicklungen der Kurse von AOL und Time Warner von 1999 bis zur Merger-Ankündigung am 10. Januar 2000.

Aus Kapitalmarktsicht wird durch M\&A-Transaktionen dann Mehrwert generiert, wenn der bereinigte Wert einer integrierten Unternehmung höher ist als der kumulierte Wert der Einzelunternehmungen. Der bereinigte Wert entspricht dabei der so genannten abnormalen Rendite, welche die Abweichung von der normalen Durchschnittsentwicklung einer Aktienrendite angibt (Cosh/Huges 1998). Mehrwerte spiegeln sich in einer höheren Marktkapitalisierung wider, welche das Resultat erwarteter zukünftiger finanzieller Rückflüsse ist.

Die Bekanntgabe der AOL-und-Time-Warner-Transaktion wurde vom Kapitalmarkt sehr positiv aufgenommen. Der Aktienkurs der kombinierten Unternehmung stieg am Tag nach der Ankündigung mit knapp 19,2 Prozent $^{7}$ weit über die Durch-

7 Die Aktienkurse von Time Warner und AOL wurden gemäß Austauschverhältnis gewichtet. 
Abbildung 2: Entwicklung der Aktienkurse von AOL und Time Warner vor der Merger-Ankündigung

\section{BAOL}

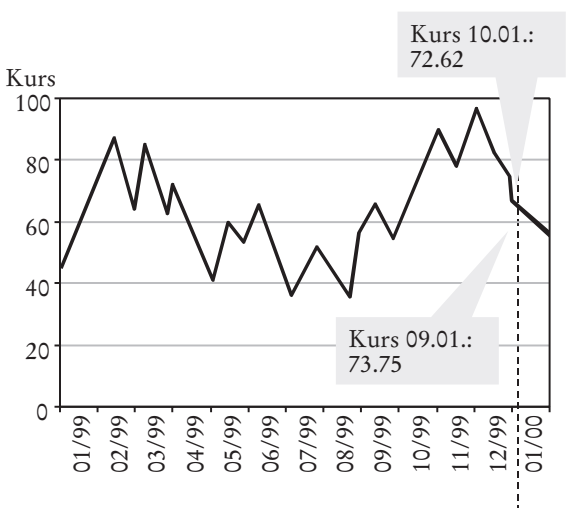

Quelle: NYSE

\section{TimeWarner}

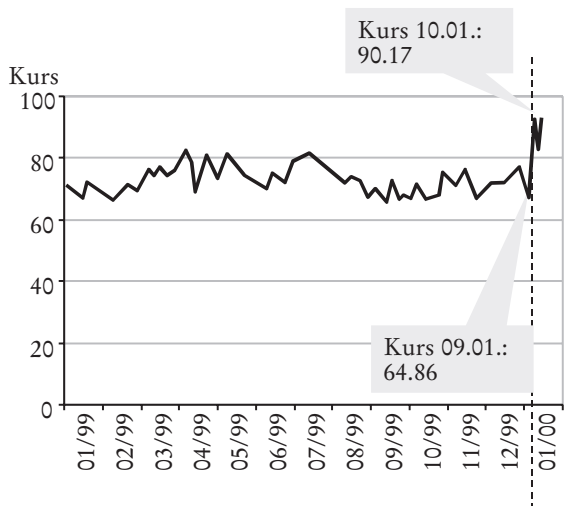

10.01.2000

schnittsentwicklung. Die Marktkapitalisierung des Unternehmens betrug USD 327 Mrd. (Waters 2000a: 1). Aus der kurzfristigen Perspektive des Kapitalmarktes ist der Merger damit als Erfolg zu werten.

Da das Geschäftsmodell von AOL auf dem Höhepunkt der Internet-Euphorie als sehr viel versprechend und vor allem skalierbar eingestuft wurde, erhielten die Aktionäre von AOL 55 Prozent an der neuen Unternehmung, die Time Warner Aktionäre mussten sich mit 45 Prozent begnügen. Trotzdem bezahlten die AOL-Aktionäre bei diesem Austauschverhältnis eine Akquisitionsprämie, indem sie auf einen proportionalen Anteil an der neuen Firma verzichteten. Aufgrund der Börsenkapitalisierung am Ankündigungstag hätten die AOL-Aktionäre rund zwei Drittel der neuen Einheit erhalten, da AOL zu diesem Zeitpunkt in etwa die doppelte Marktkapitalisierung von Time Warner aufwies. Die Kursentwicklung der beiden Einzelunternehmen folgte nach Ankündigung der Fusion einem häufig zu beobachtenden Muster. Kumuliert sind M\&As gemäß übereinstimmendem Befund zahlreicher empirischer Studien aus Sicht der Capital Market School wertstiftend. Hingegen werden die durchschnittlich leicht negativen bzw. nicht signifikant positiven abnormalen Renditen bei der Käuferunternehmung von den Gewinnen der Zielunternehmung überkompensiert (Anand/Singh 1997; Hayward/Hambrick 1997). Dies war auch bei AOL und Time Warner der Fall. Kursgewinne gingen bei der Ankündigung sowie in den ersten Monaten danach ausschließlich und deutlich an die Zielunternehmung, Time Warner, während der Kurs der Käuferunternehmung AOL nachgab, wie Abbildung 3 veranschaulicht.

Betrachtet man die Entwicklung der Marktkapitalisierung drei Jahre nach dem Merger, haben sich die Erwartungen jedoch keineswegs erfüllt. Das Synergiepotenzial wurde bei Ankündigung des AOL- und Time-Warner-Zusammenschlusses erheblich überschätzt. Kurze Zeit nach der Ankündigung platzte die Internet-Blase und die Bewertungen von Internet-Unternehmen wurden nach unten korrigiert. Auch der Aktienkurs 
Abbildung 3: Entwicklung der Aktienkurse von AOL und Time Warner nach der Merger-Ankündigung

\section{AAOL}

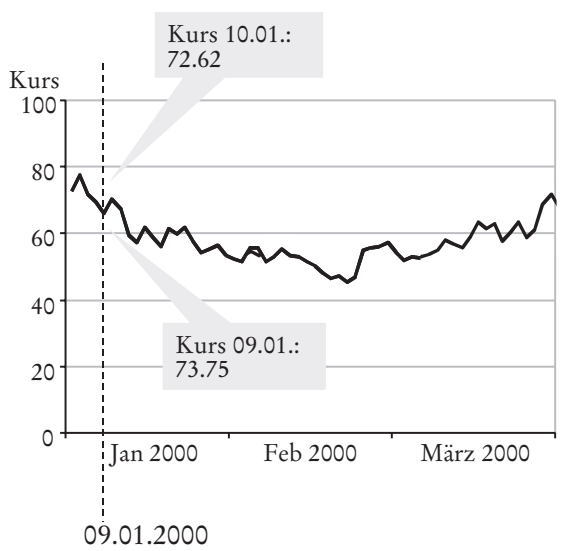

Quelle: NYSE

\section{TimeWarner}

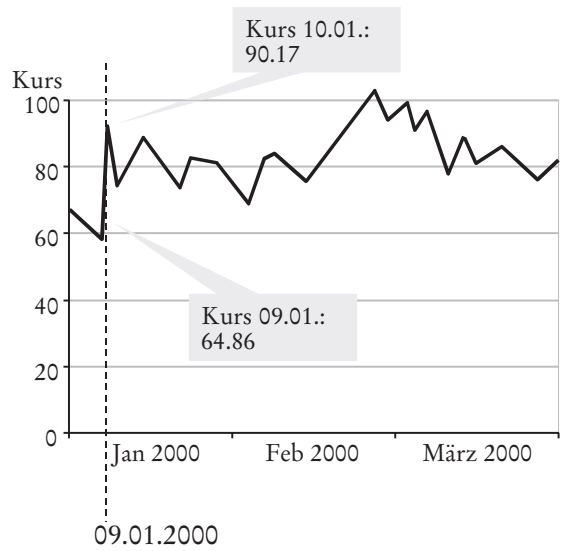

von AOL entwickelte sich nicht nur während des Jahres 2000 deutlich negativ, sondern fiel auch danach - also nach dem Vollzug der Fusion am 12. Januar 2001 - kontinuierlich weiter und stabilisierte sich erst rund zwei Jahre nach der Fusion. Dies ist gleichbedeutend mit einem markanten Post Merger Drift, d. h. der Wert der aggregierten Einheit sinkt unter den kumulierten Wert der beiden Einzelunternehmen (Pritchett et al. 1997: 127). Die Marktkapitalisierung betrug vier Jahre nach der Ankündigung noch rund USD $80 \mathrm{Mrd}^{8}{ }^{-}$rund ein Viertel des Wertes bei der Fusionsankündigung. Abbildung 4 zeigt die langfristige Kapitalmarktentwicklung des fusionierten Unternehmens.

Das Fallbeispiel zeigt eine Schwäche von kapitalmarktorientierten Ansätzen zur Beurteilung von M\&A-Transaktionen. Das Ausmaß der externen Effekte konnte während der Unterzeichnung des Mergervertrags und in der kurzen Zeit nach der öffentlichen Ankündigung kaum antizipiert und eingeschätzt werden. Aus der Sicht des Kapitalmarktes beziehen sich Wertgewinne bei M\&A-Transaktionen auf den Zeitraum der öffentlichen Ankündigung von Unternehmenszusammenschlüssen. In der Regel werden nur wenige Tage vor und nach der Transaktion betrachtet. Dahinter steht die Annahme, dass das Verhalten einer fusionierten Unternehmung voraussehbar ist und damit die aus M\&A zu erwartenden Finanzrückflüsse ex ante quantifiziert werden können. Dies wiederum erfordert einen effizienten Kapitalmarkt mit rationalem Anlegerverhalten, d. h. Anleger verwerten jederzeit alle verfügbaren Informationen über Chancen und Risiken der fusionierenden Unternehmung und können den Barwert zukünftiger Erträge abschätzen (Haspeslagh/Jemison 1991: 249). Der Fall von AOL und Time Warner zeigt deutlich die Nachteile einer ausschließlichen Kapitalmarktbetrachtung auf und unterstreicht die Bedeutung der Integrationsphase für die Wertgenerierung durch M\&A. 


\section{Abbildung 4: Langfristige Entwicklung des Aktienkurses der fusionierten Unternebmung (2000-2003)9}

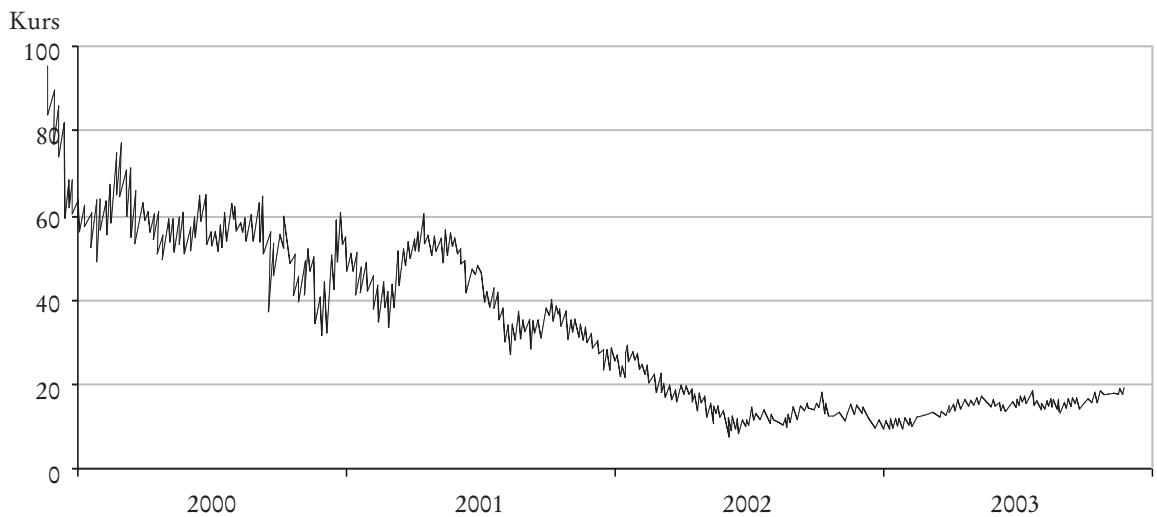

\section{Die Fusion zwischen AOL und Time Warner aus strategischer Managementsicht}

Strategische Managementansätze gehen über die Perspektive der Capital Market School hinaus. Der Erfolg von M\&A wird nicht durch einmalige, transaktionsbedingte Erlöse bestimmt, sondern durch eine verbesserte Wettbewerbssituation des neu gebildeten Unternehmens. Diese verbesserte Wettbewerbsposition führt letztlich zu nachhaltigen, strategiebedingten Renten (Haspeslagh/Jemison 1991: 25). Sie beruht auf einer strategischen Affinität der Mergerpartner. Strategischer Fit bezeichnet den Grad an strategischer Affinität zwischen zwei Fusionspartnern, d. h. inwieweit sich ihre Strategien vor dem Hintergrund der betreffenden Branche, Märkte und Technologien ergänzen und einen Beitrag zur gemeinsamen Wertschöpfung leisten. Bei einer ausgeprägten strategischen Affinität lassen sich die fusionierenden Unternehmungen nach einer einheitlichen Führungslogik steuern. ${ }^{10}$ In empirischen Fit-Untersuchungen wird analysiert, inwieweit sich Strategien von Mergerpartnern ergänzen und gegenseitig verstärken (bspw. Ellis 2000: 24; Jemison/Sitkin 1986: 146). Die Vertreter der Strategic Management School (SMS) unterstellen weiter, dass Leistungseffekte in der Integrationsphase nicht antizipiert und nicht ex ante abdiskontiert werden können. Sie berücksichtigen damit auch Vorgänge innerhalb der Unternehmung während der Integrationsphase und untersuchen deren Einfluss auf den Erfolg einer M\&A-Transaktion. Im Mittelpunkt stehen Maßnahmen mit langfristiger Wirksamkeit, welche dem bewussten Einfluss des Managements unterliegen (Larsson/Finkelstein 1999: 5). Sie sprechen in diesem Kontext auch von nachhaltiger Wertgenerierung (Value Creation), welche über eine rein transaktionsbedingte Wertgenerierung durch einen Ressourcentransfer (Asset Stripping oder Va-

9 Bis 12. Januar 2001 Aktienkurs von AOL, danach der fusionierten Unternehmung (AOL Time Warner resp. nach der Umfirmierung Time Warner)

10 Prahalad/Bettis sprechen in diesem Zusammenhang von einer so genannten Dominant general management logic (1986: 485). 
lue Capturing) hinausgeht (siehe z. B. Eccles et al. 1999). Aus Sicht der SMS ist deshalb entscheidend, wie groß der strategische Fit zwischen den Mergerpartnern ist, und wie Synergiepotenziale in der Integrationsphase durch das Management genutzt werden können (Schmidt 2000: 45). Strategisch verbundene M\&As schaffen gemäß SMS mehr Wert als strategisch unverbundene. ${ }^{11}$

Sowohl AOL als auch Time Warner sahen in ihren Geschäften einen hohen strategischen Fit mit dem jeweiligen Fusionspartner. Für AOL bestand eine hohe Affinität zum Inhaltegeschäft sowie zum Kabelbereich von Time Warner. AOL war der führende ISPAnbieter in den USA und erzielte über zwei Drittel der Erträge aus diesem Geschäft (AOL 2000: 3). Es machte deshalb für AOL strategisch Sinn, durch den Zusammenschluss eine Rückwärts-Integration in das Inhaltegeschäft vorzunehmen und an der weiteren Entwicklung des ISP-Geschäfts zu partizipieren. Um in das attraktivere Marktsegment mit höherer Wertschöpfung vorzudringen und sich innerhalb des Commodity-Markts von anderen ISPs zu differenzieren, versuchte AOL bereits vor dem Merger, mit Hilfe einer Produktbündelung von Internet-Zugang und Inhalten eine einzigartige Angebotspalette zu kreieren. ${ }^{12}$

Zudem bestand für AOL ein hoher strategischer Fit mit dem Kabelbereich von Time Warner. Bis Ende der 90er-Jahre erfolgte der Internet-Zugang fast exklusiv über Schmalband-Einwähl-Telefonleitungen (Narrowband Dial-Up Connections). Die ISPProvider bedienten sich herkömmlicher Telefonnetze, bei welchen freier Zugang bestand. Dieser freie Zugang im Telekommunikationsmarkt war für AOL von hoher Bedeutung. Der Boom des Internets im privaten Konsumenten-Bereich, zu welchem AOL mit seiner Offerte deutlich beigetragen hatte, führte jedoch zur Nachfrage nach schnelleren Verbindungen und höheren Bandbreiten. Diese konnten nicht mehr über herkömmliche Telefonleitungen angeboten werden. Für Breitband-Dienste waren Investitionen in die Netzinfrastruktur sowie in Endgeräte (aDSL End User Devices) notwendig. Zudem traten mit den TV-Kabelnetzgesellschaften, die ihre Netzwerke für Zweiweg-Kommunikation aufrüsteten, neue Wettbewerber in den Markt ein. Aufgrund der regulatorischen Rahmenbedingungen im US-amerikanischen Markt handelt es sich bei Breitband ISP-Dienstleistungen über das TV-Kabel oder die Telefonleitung jedoch - im Gegensatz zu Schmalband ISP-Dienstleistungen - nicht um eine Offerte, die ohne Koordination und Einverständnis des Netzwerkbetreibers erbracht werden kann. Der Anbieter von Mehrwertdiensten im Breitband-Bereich ist im Gegenteil von der Zustimmung des Netzwerkbetreibers abhängig, der im selben Marktsegment eigene Interessen verfolgt. Mit dem Aufkommen von Breitband-Internet und Kabelnetzbetreibern als Mitspielern war AOL deshalb gefordert, sich neu zu positionieren, wollte es nicht auf Wachstumsoptionen im Kerngeschäft verzichten. Das politische Lobbying für einen freien Zugang zum Kabelnetz und zum Breitband-Telekommunikationsmarkt blieb jedoch bis zur Ankündigung der Fusion mit Time Warner erfolglos, ebenso wie die Suche nach geeigneten Partnern. Die Kabeldivision von Time Warner, Time Warner Cable, war der zweitgrößte Anbieter im US-amerikanischen Kabelmarkt (Sjurts 2002: 367). Dazu war Time Warner als einziges kotiertes Medienkonglomerat im Kabelgeschäft tätig. Time Warner Cable war zudem der einzige große Anbieter im Kabelmarkt, der zu diesem Zeitpunkt akquiriert werden konnte (Strunck 2003: 222).

11 Siehe z. B. Ramaswamy (1997).

12 Dieses Vorhaben gelang jedoch trotz der oben erwähnten Übernahmen im Content-Bereich lediglich teilweise. 
Zusammenfassend hat aus Sicht von AOL die Fusion mit Time Warner einen hohen strategischen Fit ergeben. Erstens ermöglichte die über Time Warner realisierte Rückwärtsintegration ins Inhaltegeschäft einen Weg aus dem kostenorientierten, standardisierten ISP-Geschäft in Geschäfte mit höheren Margen und eine klare Differenzierung des Angebotes gegenüber Konkurrenzofferten. Zweitens war der Zusammenschluss mit dem Kabelgeschäft die Möglichkeit, das Kerngeschäft ISP strategisch abzusichern, sowie gleichzeitig notwendige Voraussetzung, weiter als führender Anbieter im Markt zu agieren.

Auch aus Sicht von Time Warner ergab sich ein hoher strategischer Fit mit den Geschäften von AOL. Time Warner verfügte primär über ein Portfolio aus traditionellen Mediengeschäften mit wenigen Wachstumsoptionen. Konsumenten wurden offline über Standardkanäle erreicht, und deshalb sollten Potenziale neuer Internetdistributionskanäle genutzt werden. Diese Potenziale beinhalten für ein traditionelles Medienunternehmen beispielsweise die Mehrfachverwendung von Inhalten über das Internet oder eine gezieltere Ansprache des Kunden mit Werbebotschaften, da über einzelne Kunden bspw. im Internet bessere Profile erstellt werden können als im TV-Geschäft, wo die Endkunden dem TV-Sender in der Regel nicht bekannt sind (Kolo/Vogt 2003: 253). ${ }^{13}$

Time Warner galt zwar als innovatives Unternehmen (Sjurts 2002: 369), war aber im Online-Geschäft wenig erfolgreich (Strunck 2003: 223). Eigene Initiativen, wie z.B. Pathfinder, ein Online-Meta-Brand für die Time Warner-Magazine, gelangen nicht in gewünschtem Umfang. Das Management war damit weiter auf der Suche nach Wachstumsoptionen im Internet. Somit ergab sich auch aus Sicht von Time Warner ein hoher strategischer Fit mit AOL.

Vom Kapitalmarkt wurde der relativ hohe strategische Fit zwischen AOL und Time Warner als gute Voraussetzung für einen erfolgreichen Zusammenschluss gesehen. Die Affinität der Geschäfte versprach eine solide Grundlage für die Realisierung von Mergersynergien. Insbesondere das Kosteneinsparungspotenzial in den Bereichen Operations, Management und Finanzierung wurde hoch eingestuft. ${ }^{14}$ Die Rückwärtsintegration von AOL ins Inhaltegeschäft und gleichzeitige Vorwärtsintegration von Time Warner in AOLs Onlinekanäle ließ eine starke Reduktion von aggregierten Koordinationsund Administrationskosten erwarten. Zudem sollten durch die Verteilung von Fixkosten auf einen größeren Output hohe Skalenerträge und damit operationelle Synergien erzielt werden. Beispiel dafür sind die Kosten der Inhalte-Erstellung, die durch den Verkauf über zusätzliche Kanäle, insbesondere Internet, auf einen höheren Output umgelegt werden sollten. Die Fusion bot für beide Firmen auch die Gelegenheit, sich von ineffizienten Managern oder Managementteams zu trennen und die jeweils effizienteren Führungs-, Planungs- und Kontrollsysteme vom Mergerpartner zu übernehmen. Dies bedeutete insbesondere für AOL eine Chance, Systeme, Strukturen und Prozesse zu etablieren, die in einer Start-up-Umgebung und bei stürmischem Wachstum üblicherweise nicht mit der Entwicklung des Unternehmens Schritt halten. Auf der anderen Seite konnte Time Warner den Merger nutzen, verkrustete Strukturen aufzubrechen und AOL-Manager zu Change Agents zu machen. Schließlich wurden von der Fusion von AOL und Time Warner auch finanzwirtschaftliche Synergien erwartet. Die Verschmel-

13 Eine Ausnahme bildet, selbstverständlich, das Pay-TV, wo mit den Kunden eine direkte Kundenbeziehung besteht.

14 Zur Kategorisierung von Synergien siehe Schmidt/Schettler (1999). 
zung der Geschäftsportfolios und die damit einhergehende stärkere Diversifikation versprach eine Reduktion des systematischen Risikos. Durch die zunehmende Unternehmensgröße sollte der Zugang zu günstigerem Kapital geschaffen und damit eine Senkung der Kapitalkosten erzielt werden.

Auf der Ertragsseite wurden zahlreiche Synergien ausgemacht, die vorwiegend durch das Cross-Selling von Produkten erzielt werden sollten. So wurde angestrebt, bestehenden Werbekunden zusätzliche Werbeprodukte zu verkaufen. Angedacht waren gezielte Marketingmaßnahmen bei AOL-Kunden für traditionelle Medienprodukte von Time Warner, beispielsweise für das Time Magazine, umgekehrt aber auch der gezielte Verkauf des ISP-Angebots an Abonnenten von Time Warner-Medienprodukten. Zudem sollten bestehende Kundenbeziehungen in beiden Firmen genutzt werden, um Kombinationen von verschiedenen Werbeformen (Cross-Media-Packages) aus einer Hand anzubieten.

Insgesamt wurde das ertrags- und kostenseitige Synergiepotenzial der Fusion zwischen AOL und Time Warner auf ca. USD 1,2 Mrd. geschätzt. Ertragssynergien sollten sich unmittelbar auf die Profitabilität auswirken. Schätzungen gingen davon aus, dass über 80 Prozent des zusätzlichen Umsatzes als Gewinn anfallen. Der Gesamteinfluss auf das Ergebnis der beiden Unternehmen wurde auf diese Weise auf USD 1 Mrd. geschätzt (Blodget 2000: 26).

\section{Integration von AOL und Time Warner}

Insgesamt zeigt sich, dass der Merger von AOL und Time Warner sowohl aus Kapitalmarktsicht als auch aus Sicht der Strategic Management School zur Zeit der MergerAnkündigung Erfolg versprechend erschien. Vor diesem Hintergrund ist die nachfolgende Entwicklung der fusionierten Einheit erstaunlich: AOL Time Warner konnte in den ersten zwei Jahren nach dem Zusammenschluss faktisch keines der gesetzten Mergerziele erreichen und die hohen Erwartungen keineswegs erfüllen. Im Jahr 2002 resultierte, nicht zuletzt aufgrund von Abschreibungen aus dem Merger, ein Konzernverlust von USD 99 Mrd. - der höchste Verlust in der US-amerikanischen Wirtschaftsgeschichte (Peers/Angwin 2003b: 1). Durch Probleme in der Fusions- und Integrationsphase erodierten die Vorteile des strategischen Fits zwischen AOL und Time Warner. Synergiepotenziale, die durch die Komplementarität der Geschäfte begründet waren, konnten nicht in vollem Umfang realisiert werden. Damit konnte die dem Merger zugrunde liegende Strategie nicht operativ umgesetzt werden, was sich letztendlich auch im Börsenkurs der neuen Einheit niederschlug.

Die Tatsache, dass das identifizierte Mergerpotenzial bis heute nicht realisiert werden konnte, deutet auf erhebliche Probleme in der Integrationsphase hin. Vorteile aus dem strategischen Fit zwischen AOL und Time Warner konnten offensichtlich nicht in Mergersynergien umgesetzt werden. Bei näherer Betrachtung wird deutlich, dass diese Integrationsprobleme schon mit der Strukturierung der Transaktion begannen. Eine einseitige Aufhebung des Mergervertrags wäre mit erheblichen Ausstiegskosten verbunden gewesen, denn der Merger wurde als reiner Aktientausch zu fixen Konditionen strukturiert. Beide Unternehmen verpflichteten sich zur Zahlung erheblicher Beträge, falls sie den Vertrag einseitig auflösten. Bei Time Warner wurde dieser Betrag auf USD 3,9 Mrd. festgelegt (Peers/Deogun 2000: 8). Dies entspricht knapp dem zehnfachen Wert des durchschnittlichen Nettoergebnisses der vorangegangenen fünf Jahre (Time Warner 2000b: 69). So lässt sich erklären, dass sich die Aktionäre von Time Warner ein halbes Jahr nach der Ankündigung entschieden, den Merger zu genehmigen (Peers 2000: 1), ob- 
wohl sich mit dem Platzen der Internet-Blase der Kurs der AOL-Aktie rückläufig entwickelte und aufgrund des festgelegten Umtauschwerts auch der Börsenwert von Time Warner sank. Somit war der Mergervertrag zu Konditionen geschlossen worden, welche größere externe Störfaktoren nicht mit in die Überlegungen einbezogen hatten.

Ein zweites gravierendes Problem stellte die Unsicherheit bereits im frühen Mergerstadium dar, ob der Zusammenschluss in der vorgeschlagenen Form genehmigt würde. Wie aus Post-Merger-Studien hervorgeht, ist Geschwindigkeit bei der Mergerimplementierung ein wichtiger Erfolgsfaktor (Ellis et al. 2003). Unsicherheit über den Ausgang von regulatorischen Entscheidungsprozessen können Stress und Existenzangst bei den Mitarbeitern auslösen, was sich letztendlich in Lähmungserscheinungen und defensivem Verhalten äußert (siehe Marks/Mirvis 1985; Matteson/Ivancevich 1990). Zudem ist es für die Mergerimplementierung wichtig, in einem frühen Stadium ein Momentum in der Belegschaft zu kreieren, um eigendynamische Integrationsprozesse auszulösen (Haspeslagh 2001). Zu Beginn einer Integrationsphase besteht die größte Legitimität für Veränderungen. Je länger sich Unsicherheit allerdings hinzieht, desto schwieriger werden angestrebte Veränderungen. Im Fall von AOL Time Warner dauerte es letztlich ein Jahr, bis alle regulatorischen Instanzen zustimmten, was die Integration der beiden Konzerne sichtlich verzögerte und erschwerte.

\section{Strategie}

Mit zahlreichen personellen Veränderungen auf der Ebene des obersten Managements gingen zahlreiche strategische Richtungswechsel einher. Insbesondere aus Richard Parsons' Übernahme der CEO-Position als Nachfolger von Gerald Levin resultierten strategische Neuerungen. Gerald Levin, einer der zwei Architekten der Fusion von AOL und Time Warner, verließ das Unternehmen noch im ersten Jahr nach dem Zusammenschluss. Parsons' Maßnahmen zielten auf die vollständige Umpositionierung von AOL, die Vereinfachung der Konzernstrukturen, das Wiederherstellen von Glaubwürdigkeit gegenüber den Investoren, die Nutzung von Synergien im Unternehmen sowie Investitionen in neue Technologien (Peers 2002: 1). Synergierealisierung war damit noch eines von fünf strategischen Zielen. Angestrebt wurden primär Synergien durch Einsparungen. Von Synergien auf der Ertragsseite durch Cross-Media-Offerten oder Mehrfachverwendungen von Inhalten war nicht mehr die Rede.

Die mehrfachen strategischen Richtungswechsel waren Resultat der überhasteten Strategieformulierung. Das Mergerkonzept für die neue Einheit wurde in relativ kurzer Zeit erstellt. Die Mergergespräche zwischen den Führungsgremien wurden im Oktober 1999 aufgenommen (Waters 2000a: 1); das erste Treffen zwischen Steve Case und Gerald Levin fand demnach nur drei Monate vor der Fusionsankündigung statt. Gewöhnlich resultiert ein strategisches Konzept aus einem Strategieformulierungsprozess, der sich über einen weitaus längeren Zeitraum und über mehrere Ebenen in der Organisation erstreckt (Mintzberg 1989: 34). Strategien werden sowohl durch Topdown- als auch durch Bottom-up-Prozesse erarbeitet und umgesetzt (Bower 1970; Burgelman 1983, 1994). Es existieren zahlreiche empirische Prozessuntersuchungen, die beschreiben, wie Strategieformulierungsprozesse in der Praxis verlaufen und welchen Widrigkeiten sie ausgesetzt sind (Burgelman/Grove 2002; Burgelman 1996; Mintzberg/Waters 1985). Zahlreiche Studien kommen zu dem Ergebnis, dass Top-Manager insbesondere in Mehrgeschäftsunternehmen relativ wenige Entscheidungen unmittelbar selbst treffen. Vielmehr etablieren sie einen internen Kontext (z. B. über die Gestaltung von Anreiz- und Bewertungssystemen, Organisationsstrukturen), der die 
Entscheidungsprozesse auf unterschiedlichen Hierarchieebenen steuert (Collis/Montgomery 1997: 133ff.).

Um Strategien formulieren zu können, die auch auf operativer Ebene umgesetzt werden können, ist es wichtig, Strategieformulierung und -implementierung nicht getrennt voneinander, sondern als integrierten Prozess zu betrachten (Van de Ven 1992: 169; Pettigrew 1992: 6). Feedback über Probleme bei der Implementierung vorgängiger Strategiekonzepte sind wichtige Informationen, die in die Formulierung einer neuen Strategie eingehen müssen. Dementsprechend ist ein neues Strategiekonzept auch stark von vergangenen Prozessen innerhalb der beteiligten Firma geprägt. Die Evolutionstheorie spricht in diesem Zusammenhang auch von Pfadabhängigkeiten für die Unternehmensentwicklung (Baum 1996; Nelson/Winter 1982).

In einer Mergersituation wie bei AOL und Time Warner kommt es nun bei der Festlegung des Mergerkonzepts zu einer fast vollständigen Entkopplung von Strategieformulierung und -implementierung. Um nicht Gegenstand öffentlicher Spekulationen zu werden, sehen sich Mergerpartner gezwungen, den Verhandlungs- und Einigungsprozess möglichst kurz und unter Ausschluss der Öffentlichkeit zu halten. Dementsprechend müssen gegenseitige Treffen geheim gehalten werden, und es können nur relativ wenige Personen in die Verhandlungen involviert werden. Zudem bringt die Mergersituation das Problem mit sich, dass zuvor unabhängige Managementteams einen gemeinsamen Strategieformulierungsprozess gestalten müssen, welcher Partikularinteressen vielfältiger Anspruchsgruppen berücksichtigt.

\section{Kultur}

Neben dem strategischen Fit bildet der kulturelle Fit der sich verbindenden Organisationen einen wichtigen Faktor für die Einschätzung der Realisierbarkeit des Mergerkonzepts (Ellis et al. 2003). Eine hohe Kulturkompatibilität erleichtert grundsätzlich die operative Zusammenführung der Mergerpartner. Allerdings lässt sich die Integration von Unternehmenskulturen nicht am Reißbrett entwerfen und dann wie geplant implementieren. Vielmehr handelt sich bei der Zusammenführung von Unternehmen um einen komplexen Verschmelzungsprozess unterschiedlicher Normen, Werte und Wissensbestände. Daher erscheint es notwendig, dass neben dem strategischen Fit im Vorfeld von Megafusionen - und insbesondere von Mergers of Equals - analysiert werden sollte, inwieweit unterschiedliche Wertvorstellungen, Eigenschaften, Fähigkeiten und Erfahrungen Integration und Erfolg eines Zusammenschlusses fördern oder beeinträchtigen können.

Um den Übereinstimmungsgrad der Unternehmenskulturen zu bestimmen und mögliche Problemfelder für die Integration zu identifizieren, sollten die bestehenden Unternehmenskulturen inklusive ihrer Untersysteme (Subkulturen) systematisch erfasst und hinsichtlich ihres Verankerungsgrades und Übereinstimmungsmaßes analysiert werden. Für die multidimensionale Ist-Erfassung von Unternehmenskulturen existieren zahlreiche Ansätze in der strategischen Managementliteratur (siehe z. B. Meffert/Hafner/Poggenpohl 1990; Rühli 1991; Treichler 1995). Im Wesentlichen geht es hierbei um die systematische Erstellung von Profilen, wobei Kulturmerkmale, wie z. B. Mitarbeiterorientierung, Leistungsorientierung, Konkurrenzorientierung oder Mitspracherecht der Mitarbeiter, Informations- und Kommunikationsbeziehungen, Rituale im Umgang mit Kunden, Gestaltung der Firmengebäude etc., nach ihrer Ausprägungsform anhand von Likert-Skalen (z. B. über ein Kontinuum von stark bis schwach) klassifiziert werden. 
Im nächsten Schritt gilt es, eine Soll-Kultur auf gesamtkultureller Ebene und auf Ebene der Subkulturen für das neue Gebilde zu definieren, welche in Einklang mit strategischen und strukturellen Zielsetzungen des Zusammenschlusses steht. Auf Basis einer Lückenanalyse zwischen Ist- und Soll-Kultur kann zumindest das Ausmaß an potenziellen Integrationsbarrieren und deren Einfluss auf die Realisierbarkeit des Mergerkonzepts abgeschätzt werden. Zudem lassen sich gezielte Maßnahmen zur Vermeidung von erwarteten Kulturkonflikten (z.B. durch Festlegung von Verhaltensregeln) sowie zur Verstärkung gewünschter bzw. zur Veränderung unerwünschter Kulturelemente ableiten, die dann wichtige Bestandteile der Integrationsphase bilden.

Dieses skizzierte generische Vorgehen zur Analyse des kulturellen Fits zwischen Mergerpartnern ist natürlich nicht immer in dieser Form sinnvoll. Im Rahmen des AOL- und Time-Warner-Mergers war z. B. auch ohne eine systematische Erfassung der Kulturmerkmale offensichtlich, dass zwei sehr unterschiedliche Unternehmenskulturen aufeinander trafen. Hier stellte sich weniger die Frage, wie unterschiedlich die Kulturen waren, sondern eher $o b$ diese Unterschiedlichkeit bewusst erhalten werden sollte oder nicht (vgl. Haspeslagh/Jemison 1991: 143). Die kulturellen Integrationsprobleme bei der Fusion von AOL und Time Warner wurden auch durch die Probleme der Transaktionsstruktur sowie der Unsicherheit über den Ausgang der kartellrechtlichen Prüfung des Mergers genährt und äußerten sich in Machtkämpfen des Top-Managements. Daraus resultierten mitunter zahlreiche personelle Veränderungen. Zunehmend verließen frühere AOL-Manager das Unternehmen als Reaktion darauf, dass Time-Warner-Manager für die Besetzung von Vakanzen bevorzugt wurden. Im Januar 2003, drei Jahre nach der Merger-Ankündigung und zwei Jahre nach dem erfolgten Zusammenschluss, kündigte Chairman Steve Case seinen Rücktritt als letztes Mitglied des ehemaligen AOL-Top-Managements an. Wenige Monate nach seinem Rücktritt beschloss der Konzern die Umbenennung von AOL Time Warner zum alten Firmennamen des übernommenen Unternehmens, TimeWarner. Mit seinem Abgang und der Freisetzung von weiteren Schlüsselpersonen aus der ehemaligen AOL ging ein Teil von AOLs unternehmerischen Werten, wie z. B. Kostenfokus, Risikofreude und Tempo, die auf das fusionierte Unternehmen übertragen werden sollten, verloren.

\section{Fazit und Ausblick}

Aufgrund der oben dargestellten Rahmenbedingungen und Entwicklungen ist es wenig erstaunlich, dass bei AOL und Time Warner ein Mergerkonzept erarbeitet wurde, das sich nicht in geplanter Weise umsetzen ließ. Die Transaktion machte zwar konzeptionell Sinn, was die Steigerung der Börsenkurse nach der Ankündigung dokumentierte. Infolge des Zeitdrucks sowie des mangelnden Rückgriffs auf funktionale Spezialisten mit dem notwendigen Detailwissen blieb das Mergerkonzept aber zu abstrakt für eine zügige Umsetzung der Integration. Um zum Zeitpunkt der Fusionsankündigung die Durchführbarkeit des Mergers besser beurteilen zu können, wäre das Detailwissen und die Erfahrung des operativen Managements notwendig gewesen. ${ }^{15}$

15 Erschwerend für die Integration kam hinzu, dass es sich bei den beabsichtigten Strategien um völlig neue Ansätze handelte, die zu diesem Zeitpunkt noch in keinem vergleichbaren Medien-, Unterhaltungs- oder Internetunternehmen umgesetzt worden waren. Pfadabhängigkeiten, vor allem beim eher traditionell ausgerichteten Unternehmen Time Warner, wurden in den Verhandlungen nicht genügend gewichtet bzw. unterschätzt. 
Integrationsbarrieren wurden bei der Fusion von AOL und Time Warner offensichtlich unterschätzt bzw. Synergiepotenziale überschätzt. Zudem lag ein Problem in der Operationalisierung des Mergerkonzepts. Der Operationalisierbarkeit des Mergerkonzepts kommt insofern große Bedeutung zu, da es neben der langfristigen Ausrichtung der neuen Einheit gleichzeitig auch die Leitlinien für die Integration der beteiligten Unternehmen vorgibt. So sollte neben dem Synergiepotenzial auch ein Faktor für die Realisierbarkeit des Mergerkonzepts ermittelt werden, welcher z. B. das Synergiepotenzial von Mergerkandidaten relativieren könnte.

Um diese Einschätzung vornehmen zu können, ist es allerdings nicht ausreichend, den strategischen Fit zum Zeitpunkt des Mergers zu evaluieren. Vielmehr ist es erforderlich, dem Merger vorgelagerte Strategieformulierungsprozesse und Strategieimplementierungsprozesse zu identifizieren und deren Implikationen für den gemeinsamen Strategieprozess der neuen Einheit abzuleiten. Bei einem Megamerger wie AOL und Time Warner werden keine grundsätzlich neuen bzw. neuartigen Strategieprozesse ausgelöst (Schmidt 2000). Die strategische Ausrichtung der neuen Unternehmung ist vielmehr in seinen tragenden Elementen durch die Strategieprozesse der zuvor unabhängig agierenden Firmen determiniert („History matters“, vgl. Mintzberg 1977: 17; Kieser 1994). Die Analyse dieser vorausgehenden Strategieprozesse lässt somit wichtige Voraussagen über das zukünftige Verhalten fusionierter Großunternehmungen zu und ermöglicht eine fundiertere Einschätzung der Realisierungschancen eines Mergers.

Die Probleme bei AOL Time Warner sind in Bezug auf die Entkopplung von Strategieformulierungs- und Implementierungsprozess letzten Endes kein Sonderfall. Eine ähnliche Problematik ergibt sich generell für große Unternehmenszusammenschlüsse und lässt sich aufgrund der besonderen Situation eines Mergers (z. B. Geheimhaltungspflichten) auch nicht grundsätzlich vermeiden. Bisherige Analysemethoden sind, wie das Beispiel von AOL Time Warner zeigt, nicht hinreichend, um die Erfolgswahrscheinlichkeit eines Mergers vorauszusagen. Hohe Synergiepotenziale und ein strategischer Fit zwischen den Mergerpartnern sind a priori kein Garant für den Mergererfolg. Häufig kann aufgrund von Integrationsbarrieren nur ein Teil der kalkulierten Synergien realisiert werden (Haspeslagh/Jemison 1991; Harrison/Hitt 1991).

AOL Time Warner war die erste Megafusion zwischen einem traditionellen Medienunternehmen und einer Internetfirma, welcher als Zusammenschluss gleichberechtigter Partner arrangiert wurde. Bei Aufsehen erregenden Transaktionen in der Medienindustrie in der Vergangenheit (wie z. B. von Sony und Columbia Pictures, Disney und Capital Cities/ABC oder Time Warner und Turner Broadcasting Systems) handelte es sich stets um klassische Akquisitionen. Auch Übernahmen von Internet-Unternehmen lassen kaum Vergleiche zur Fusion von AOL und Time Warner zu. Entweder haben traditionelle Medienunternehmen neue Firmen wie Internet-Startups übernommen oder Internetfirmen haben kleinere Konkurrenten gekauft (z. B. Kauf von Netscape durch AOL). Deshalb lässt sich der Zusammenschluss nicht mit anderen Fusionen in der Medienindustrie vergleichen. Vielmehr bietet sich ein Vergleich mit Zusammenschlüssen ähnlicher Größenordnung aus anderen Branchen an. Bei diesem Vergleich zeigt sich, dass mit dem Zusammenschluss von AOL und Time Warner auch Probleme einhergingen, die nicht speziell mit der Industrie zusammenhängen, sondern vielmehr grundsätzlich mit Megafusionen verbunden sind.

Die Fusion von AOL und Time Warner war beispielhaft für Zusammenschlüsse der so genannten fünften Mergerwelle, welche von 1992 bis 2001 andauerte (siehe Böckli 2003; Kleinert/Klodt 2000). Die Fusionswelle der 90er-Jahre war geprägt durch große, freundliche, horizontale, verwandte und aktienfinanzierte Unternehmenszusammen- 
schlüsse (wie eben der von AOL und Time Warner). Dabei ist es auch interessant zu beobachten, dass zahlreiche dieser Megafusionen als Merger of Equals gestaltet wurden (siehe z. B. Daimler Benz/Chrysler, Thyssen/Krupp, UBS/Bankverein, Sandoz/Ciba). Ein solcher Zusammenschluss unter Gleichen verspricht unmittelbar wirtschaftliche und psychologische Vorteile (Schmidt 2001). Durch den Aktientausch im Sinne einer Kombination müssen keine Übernahmeprämien gezahlt werden. Zudem kann mit geringeren Integrationsbarrieren und -kosten gerechnet werden, da betroffene Anspruchsgruppen eher für einen Zusammenschluss von zwei gleichberechtigten Unternehmen gewonnen werden können, als wenn eine Unternehmung von der anderen übernommen wird.

Betrachtet man indessen die Umsetzung von Mergers of Equals in der Unternehmenspraxis, dann fällt auf, dass die angenommene Reduktion der Integrationsbarrieren nicht automatisch eintritt. Neben der Tatsache, dass sich die vertraglich vereinbarte $\mathrm{Pa}$ rität nicht ohne Weiteres im Integrationsprozess aufrechterhalten lässt, entstehen vor allem Probleme bei der Verschmelzung zweier unterschiedlicher Firmenkulturen. Ähnlich wie bei Daimler/Chrysler, wo deutsche und amerikanische Managementstile und Geschäftsgebaren aufeinander stießen und große Reibungsverluste innerhalb der Mergerintegration verursachten, kam es auch bei Fusion von AOL und Time Warner zu einem „Kulturschock“. Zwar handelte es sich nicht um einen transatlantischen Zusammenschluss, aber es trafen die kostenfokussierte, gewinn- und technologieorientierte Kultur von AOL und die umsatzorientierte, auf Produktinnovationen fokussierte und auf konservative Werte ausgerichtete Unternehmenskultur von Time Warner aufeinander (Sjurts 2000). Dass es schließlich den beiden Firmen nicht gelang, die Kulturen im Sinne eines Merger of Equals erfolgreich zusammenzuführen, hatte sicherlich auch negative Auswirkungen auf den Merger-Erfolg.

Diese Annahme wird gestützt durch verhaltenswissenschaftlich ausgerichtete Organisationsliteratur, wonach kulturelle Divergenzen die Erfolgsaussichten eines aus Produkt-, Markt- oder Finanzperspektive viel versprechenden Zusammenschlusses trüben. Die durch das Aufeinandertreffen unterschiedlicher Unternehmenskulturen entstehenden Konflikte in der Integrationsphase können zu Abgängen oder Leistungseinbußen wichtiger Mitarbeiter führen und somit nachhaltigen Schaden verursachen (Larsson/Finkelstein 1999). Diese Annahmen werden durch die Ergebnisse einer empirischen Analyse von über 100 Megafusionen in den 90er-Jahren gestützt, die belegen, dass die erwarteten Reibungsverluste durch das Zusammenführen unterschiedlicher Unternehmenskulturen oftmals größer waren als vorgesehen (Kleinert/Klodt 2000: 97).

Folglich ist neben einer strategischen auch die kulturelle Übereinstimmung der beteiligten Unternehmen Voraussetzung für den Erfolg von Unternehmenszusammenschlüssen (Buono et al. 1988; Malekzadeh/Nahavandi 1990). Eine hohe Kulturkompatibilität wirkt sich nicht nur positiv auf die Motivation der Mitarbeiter aus und begünstigt damit die Personalintegration, sondern erleichtert die gegenseitige Kommunikation, welche wiederum Voraussetzung für die Realisierung der Synergieziele ist (Cartwright/Cooper 1993).

Der Erfolg einer Megafusion hängt neben der strategischen und kulturellen Passung der Mergerpartner und dem Management der Integration aber schlussendlich immer auch von unbeeinflussbaren Umweltbedingungen ab. Das Ausmaß der durch einen Merger generierten Zusatzerlöse (wie z.B. durch ein kombiniertes Produktangebot) wird mitunter maßgeblich durch gesamtwirtschaftliche Faktoren beeinflusst. Dabei gelten Strukturwandel, Deregulierung sowie Globalisierung häufig als wesentliche Einflussfaktoren, welche sich zum Teil gegenseitig bedingen und verstärken (Böckli 2003; 
Kleinert/Klodt 2000). Die Ertragskraft der fusionierenden Unternehmen kann durch das gesamtwirtschaftliche Klima allerdings in ganz unterschiedlicher Weise betroffen sein. Erhebliche Differenzen ergeben sich insbesondere dann, wenn die Fusionspartner unterschiedliche Erlösquellen aufweisen wie im Fall von AOL und Time Warner. Die Erlöse von AOL entwickelten sich - wie zuvor gezeigt werden konnte - infolge der abrupt endenden Internet-Euphorie nach dem Zusammenschluss wesentlich schlechter als die von Time Warner. Daraus resultierte eine nicht prognostizierte drastische Verschiebung der relativen Bedeutsamkeit beider Erlösbereiche, der Machtpositionen, der Motivation der Mitarbeiter beider Bereiche und damit letztendlich auch der Kohärenz und Leistungskraft. Die Prognostizierbarkeit gesamtwirtschaftlicher Veränderungen gestaltet sich in der Unternehmenspraxis zwar als schwierig, aber im Zuge von Megafusionen ist es aufgrund ihrer Tragweite unabdingbar, unsystematische Risiken zu prüfen und Anspruchgruppen ausreichend darüber zu informieren.

\section{Literaturverzeichnis}

Anand, J./Singh H. (1997): Asset Redeployment, Acquisitions and Corporate Strategy in Declining Industries. In: Strategic Management Journal, Vol. 18 (Summer Special Issue), S. 99-118.

AOL (2000): America Online, Inc. 2000 Annual Report to Shareholders. Dulles.

Baum, J. A. (1996): Organizational ecology. In: Clegg, S. R./Hardy, W./Nord, W. R. (Hrsg.): Handbook of organization studies. London et al., S. 77-114.

Blodget, H. (2000): You've got upside - America Online/Time Warner. Merrill Lynch Research, 23.02.2000, New York.

Böckli, M. A. (2003): Megafusionen: Erfolgs- und Prozessaspekte. Bern.

Bower, J. L. (1970): Managing the resource allocation process. Boston.

Buono, A. F./Bowditch J. L. et al. (1988): The cultural dynamics of transformation. In: Kilman, R./Covin, T. (Hrsg.): Corporate transformation: Revitalizing organizations for a competitive world. San Francisco, S. 497-522.

Burgelman, R. A. (1983): A model of the interaction of strategic behavior, corporate context, and the context of strategy. In: Academy of Management Review, Jg. 8, S. 61-70.

Burgelman, R. A. (1994): Fading memories - A process study of strategic business exit in dynamic environments. In: Administrative Science Quarterly, Jg. 39, S. 24-56.

Burgelman, R. A. (1996): A process model of strategic business exit - implications for an evolutionary perspective on strategy. In: Strategic Management Journal, Jg. 17 (Special Issue Summer), S. 193-214.

Burgelman, R. A./Grove, A. S. (2002): Strategy is destiny: How strategy making shapes a company's future. Stanford, CA.

Cartwright, S./Cooper, G. L. (1993): The role of culture compatibility in successful organizational marriage. In: Academy of Management Executive, Jg. 7, S. 57-70.

Collis, D. J./Montgomery C. A. (1997): Corporate strategy: Resources and the scope of the firm. Irwin.

Cosh, A./Hughes, A. (Hrsg.) (1998): Takeovers - Volume II, The International Library of Management. Aldershot.

Eccles, R. G., Lanes, K. L./Wilson, T. C. (1999): Are you paying too much for that acquisition? In: Harvard Business Review, Vol. 77, S. 136-147.

Ellis, K. M. (2000): Outcomes in related acquisitions of similar-sized firms: The effects of integration approach and process management. Tallahassee.

Ellis, K. M. /Weber Y./Raveh, A. (2003): Exploring differences in synergy potential and implementation activities across M\&A integration approaches. Unpublished working paper, Michigan State University, Department of Management.

Grimes, C. (2001): The odd couple make their preparations for the big day. In: Financial Times, 12.01.2001, S. 15. 
Harrison, J. S./Hitt, M. A. et al. (1991): Synergies and post-acquisition performance: Differences versus similarities in resource allocations. In: Journal of Management, Jg. 17, S. 173-190.

Haspeslagh, P. C. (2001): Maintaining momentum in mergers and acquisitions. Working Paper, INSEAD, Fontainbleau.

Haspeslagh, P. C./Jemison, D. B. (1991): Managing acquisitions. New York.

Hayward, M. L. A./Hambrick D. C. (1997): Explaining the Premiums Paid for Large Acquisitions: Evidence of CEO Hubris. In: Administrative Science Quarterly, Vol. 42, S. 103-127.

Huber, P. (2000): Death of old media. In: The Wall Street Journal, 11.01.2000, S. 26.

Jemison, D. B. / Sitkin S. B. (1986): Corporate acquisitions. A process perspective. In: Academy of Management Review, Vol. 11, S. 145-163.

Kieser, A. (1994): Why organizational theory needs historical analyses - and how this should be performed. In: Organization Science, Jg. 5, S. 608-620.

Klein, A. (2003): Stealing time: Steve Case, Jerry Levin, and the collapse of AOL Time Warner. New York.

Kleinert, J./Klodt, H. (2000): Megafusionen: Trends, Ursachen und Implikationen. Tübingen.

Klinkenborg, V. (2000): Does it matter who owns what? In: The New York Times, 13.01.2003, S. 32 .

Kolo, C./Vogt, P. (2003): Strategies for growth in the media and communication industry: Does size really matter? In: The International Journal on Media Management, Vol. 5 (4), S. 252-262.

Larsson, R./Finkelstein S. (1999): Integrating strategic, organizational and human resource perspectives on mergers and acquisitions: A case survey of synergy realization. In: Organization Science, Jg. 10, S. 1-26.

Levin, G. (2000): Dear shareholders. In: Keyword: Time Warner 2000. Annual Report to Shareholders 1999. New York, S. 3-5.

Levin, G./Case, S. (2000): AOL Time Warner: A new kind of enterprise. In: Keyword: Time Warner 2000. Annual Report to Shareholders 1999. New York, S. 7.

Malekzadeh, A. R./Nahavandi, A. (1990): Making mergers work by managing cultures. In: The Journal of Business Strategy, Jg. 2, S. 55-57.

Marks, M. L./Mirvis, P. (1985): Merger syndrome: Stress and uncertainty. In: Mergers and Acquisitions, Jg. 20, S. 50-55.

Matteson, M. T./Ivancevich, J. M. (1990): Mergers and Acquisition Stress: Fear and Uncertainty. In: Quick, J. C./Hess, R. E. et al. (Hrsg.): Career stress in changing times. New York, S. 139-158.

Meffert, H./Hafner, K./Poggenpohl, M. (1990): Unternehmenskultur und Unternehmensführung - Ergebnisse einer empirischen Untersuchung. In: Simon, H. (Hrsg.): Herausforderung Unternehmenskultur, USW-Schriften für Führungskräfte, Band 17. Stuttgart, S. 47-63.

Mintzberg, H. (1977): Strategy formulation as an historical process. In: International Studies of Management \& Organization, Jg. 7, S. 28-42.

Mintzberg, H. (1989): Mintzberg on management. Inside our strange world of organizations. New York.

Mintzberg, H./Waters, J. A. (1985): Of strategies, deliberate and emergent. In: Strategic Management Journal, Jg. 6, S. 257-272.

Munk, N. (2003): Steve Case, genius. In: The New York Times, 15.01.2003, S. 15.

Nelson, R. R./Winter, S. G. (1982): An evolutionary theory of economic change. Cambridge, MA.

Peers, M. (2000): Time Warner holders stand by deal with AOL. In: The Wall Street Journal, 06.12.2002, S. C1.

Peers, M. (2002): In shift, AOL Time Warner to de-emphasize 'convergence'. In: The Wall Street Journal, 13.05.2002, S. B1.

Peers, M./Angwin, J. (2003a): Steve Case quits as AOL chairman under pressure - Time Warner investors blames him for sell-off in stock after big merger. In: The Wall Street Journal, 13.01.2003, S. A1.

Peers, M./Angwin, J. (2003b): AOL reports record annual loss and says Ted Turner will resign. In: The Wall Street Journal, 30.01.2003, S. A1.

Peers, M./Brown, K. (2003): AOL's winners and losers - three big media investors demonstrate their clout in ousting chairman Case. In: The Wall Street Journal, 14.01.2003, S. B1. 
Peers, M./Deogun, N. (2000): Case's top America Online executives still will report to him after merger. In: The Wall Street Journal, 17.01.2000, S. B8.

Pettigrew, A. M. (1992): The character and significance of strategy process research. In: Strategic Management Journal, Jg. 13, S. 5-16.

Prahalad, C. K./Bettis, R. A. (1986): The dominant logic - a new linkage between diversity and performance. In: Strategic Management Journal, Jg. 7(6), S. 485-501.

Pritchett, P./Morison, D. et al. (1997[1985]): After the merger: The authoritative guide for integration success. New York.

Ramaswany, K. (1997): The performance impact of strategic similarity in horizontal mergers. In: Academy of Management Journal, Jg. 40, S. 697-717.

Rühli, E. (1991): Unternehmungskultur - Konzepte und Methoden. In: Rühli, E./Keller, A. (Hrsg.): Kulturmanagement in schweizerischen Industrieunternehmen. Bern, S. 11-49.

Schmidt, S. L. (2000): Megamerger in der pharmazeutischen Industrie. Ein Beitrag zur Strategieprozessforschung. Bern.

Schmidt, S. L. (2001): Merger of Equals - Idee und Wirklichkeit. In: Die Betriebswirtschaft, Jg. 61, S. 601-614.

Schmidt, S. L./Schettler, M. (1999): Ziele von Unternehmungszusammenschlüssen: Theorie und Praxis. In: Zeitschrift Führung und Organisation, Jg. 68(6), S. 312-317.

Sjurts, I (2002): Strategien in der Medienbranche: Grundlagen und Fallbeispiele. Wiesbaden.

Sjurts, I. (2000): Die Fusion AOL - Time Warner: Eine strategietheoretische Erklärung. In: Zeitschrift Führung Organisation, Jg. 69(3), S. 128-138.

Strunck, A.W. (2003): Strategien für Mergers \& Acquisitions in der Medienindustrie. Dissertation der Universität St. Gallen (HSG). St. Gallen.

Time Warner (2000a): Keyword: Time Warner 2000. Annual Report to Shareholders 1999, New York.

Time Warner (2000b): Time Warner Inc. 1999 Financials. Keyword: TW Financials. New York.

Treichler, C. (1995): Kulturbewusste Unternehmungsführung - Entwicklung eines Problemlösungskonzepts unter besonderer Berücksichtigung der Instrumente der Erfassung, Beurteilung und Gestaltung der Unternehmungskultur. Bern.

Van de Ven, A. H. (1992): Suggestions for studying strategy process: A research note. In: Strategic Management Journal, Jg. 13, S. 169-188.

Waters, R. (2000a): Media titans in USD 327bn merger. In: Financial Times, 11.01.2000, S. 1.

Waters, R. (2000b): A new media world. In: Financial Times, 11.01.2000, S. 22. 